\title{
RECUPERACIÓN DE ÁREAS DAÑADAS POR LA MINERÍA EN LA CANTERA LOS GUAOS, SANTIAGO DE CUBA, CUBA
}

\author{
ALEXIS MONTES DE OCA-RISCO ${ }^{1}$, MAYDA ULLOA-CARCASSÉS ${ }^{2}$
}

Recibido el 19 de marzo de 2013 y aprobado el 28 de mayo de 2013

\section{RESUMEN}

La provincia de Santiago de Cuba tiene concesionados 10 yacimientos donde se explotan geo-recursos destinados a la producción de materiales para la construcción. Las áreas dañadas por la explotación de materiales de la construcción en la provincia ocupan alrededor de 43 ha, lo cual provoca un fuerte impacto al medio ambiente. Por tal razón se propuso como Objetivo: elaborar un procedimiento para recuperar las áreas minadas en las canteras de materiales de construcción de Santiago de Cuba que permita lograr una minería responsable. En la metodología: se aplicaron métodos científicos que permitieron el análisis de los factores que influyen en la elección de los usos de recuperación en áreas minadas de la zona de estudio. Alcanzando como resultado: un procedimiento que consta de cinco etapas principales. $Y$ como conclusión: se demostró que se puede mejorar la calidad ambiental, social y económica en las zonas afectadas por la actividad minera.

\section{PALABRAS CLAVE}

Canteras, minería responsable, medio ambiente, recuperación, impacto ambiental.

\section{RESTORATION OF AREAS AFFECTED BY MINING IN THE GUAOS QUARRY, SANTIAGO DE CUBA, CUBA}

\section{ABSTRACT}

The province of Santiago de Cuba has 10 mining deposits franchised where geo-resources for production of building materials are exploited. The areas that are damaged by the exploitation of construction materials in the province occupy about 43 ha, which is provoking a strong environmental impact. For this reason the proposed objective of this research was: To develop a method to restore the areas affected by mining in the building materials quarries in Santiago de Cuba which allowed achieving responsible mining. Methodology: scientific methods were applied which allowed the analysis of the factors that influence in the selection of the restoration uses in mining areas in the study zone. Results: A process consisting of five major stages. Conclusion: it was demonstrated that it is possible to improve the environmental, social and economic quality in the areas affected by mining activities.

\section{KEY WORDS}

Quarries, responsible mining, environment, restoration, environmental impact. 


\section{INTRODUCCIÓN}

La minería, actividad vinculada a la explotación de los recursos naturales, es responsable de grandes alteraciones negativas sobre el medio ambiente y en particular, de la degradación de los suelos. Pero también, a través de ella, es que se obtienen todos los recursos necesarios para el desarrollo de la humanidad y el bienestar social del hombre.

Parrota y Knowles (2001) plantean que el aumento de la capacidad humana de transformar el entorno natural, ha originado un desequilibrio entre los deterioros ocasionados y la capacidad de recuperación del medio frente a los mismos. Pero a la vez, es evidente que no se puede prescindir de la minería porque es la actividad básica dedicada a la obtención de los georecursos para el abastecimiento a la sociedad de las materias primas necesarias para mejorar su calidad de vida, su progreso y su destino. Sin embargo, la conciencia que se tiene hoy de la limitación de los recursos naturales, así como la de los diversos elementos que componen los ecosistemas, obligan a solucionar los problemas de la demanda de materias primas en equilibrio con la conservación de la naturaleza, de manera que permita salvaguardar el patrimonio que representa el medio y los recursos naturales para poder legarlos a las futuras generaciones.

La extracción de materiales a cielo abierto es un tipo de disturbio antrópico que afecta todos los factores del ecosistema (vegetación, fauna, suelos, etc.), las geoformas del terreno y las condiciones microclimáticas. Las áreas de extracción de materiales, en muchos casos, se encuentran localizadas en los límites de las ciudades. Cuando la extracción de materiales se realiza irracionalmente sin una planeación de la explotación, los problemas que genera después del abandono son muy graves debido a que los taludes quedan inestables y se producen deslizamientos, que a su vez pueden causar pérdidas de vidas humanas. Otros problemas que crea una explotación no planeada son la pérdida del suelo superficial, contaminación de las aguas superficiales, emisiones atmosféricas de polvo y la emisión de ruido (Carbonell, 2003).

En Cuba existen regulaciones ambientales para toda actividad que genere deterioro al entorno. En este sentido, se ha instituido la Evaluación de Impacto Ambiental como uno de los niveles fundamentales de la gestión ambiental y se ha promulgado la Ley 81 (Ley del Medio Ambiente), que establece la obligación de minimizar o mitigar los efectos negativos al medio ambiente; así como la Ley 76 de Minas que plantea en su artículo 41 que los concesionarios están obligados a preservar adecuadamente el medio ambiente y las condiciones ecológicas del área, y deben elaborar estudios y planes para prevenir, mitigar, controlar, rehabilitar y compensar el impacto derivado de la actividad minera, tanto en dicha área como en las áreas y ecosistemas vinculados a aquellos que puedan ser afectados.

El incumplimiento de lo establecido en los proyectos mineros en cuanto a legislación ambiental, según Quesada (2008) ha propiciado que muchas veces los terrenos explotados no sean adecuadamente rehabilitados al cesar las labores de minería.

La provincia de Santiago de Cuba tiene concesionados 10 yacimientos donde se explotan geo-recursos destinados a la producción de materiales para la construcción, que han ocasionado una afectación al medio ambiente 
debido al fuerte impacto de las áreas dañadas que ocupan alrededor de 43 ha en toda la provincia.

El 27\% del área que se encuentra afectada en la provincia por la minería pertenece a la Cantera Los Guaos. Hasta el momento no se cuenta con un instrumento eficiente que recoja de forma integral los pasos a seguir en la recuperación de los espacios dañados por la minería, y por esta razón es importante establecer un procedimiento para la recuperación de estas áreas.

En este trabajo se propone un procedimiento para la recuperación de las áreas dañadas por la minería y se aplica en la Cantera Los Guaos perteneciente a la Industria de Materiales de la Construcción de la provincia de Santiago de Cuba.

\section{Caracterización de la Industria de Materiales de Construcción de Santiago de Cuba}

La Industria de Materiales de Construcción de Santiago de Cuba en la actualidad tiene en explotación 10 yacimientos (Siboney, Yarayabo, Mucaral, Juraguá, Dos Palmas, Maceira Vaquería, Los Dorados, Baitiquirí, Dorados II y Los Guaos) (Figura 1), los cuales están destinados a satisfacer las crecientes necesidades que se imponen en toda la provincia.

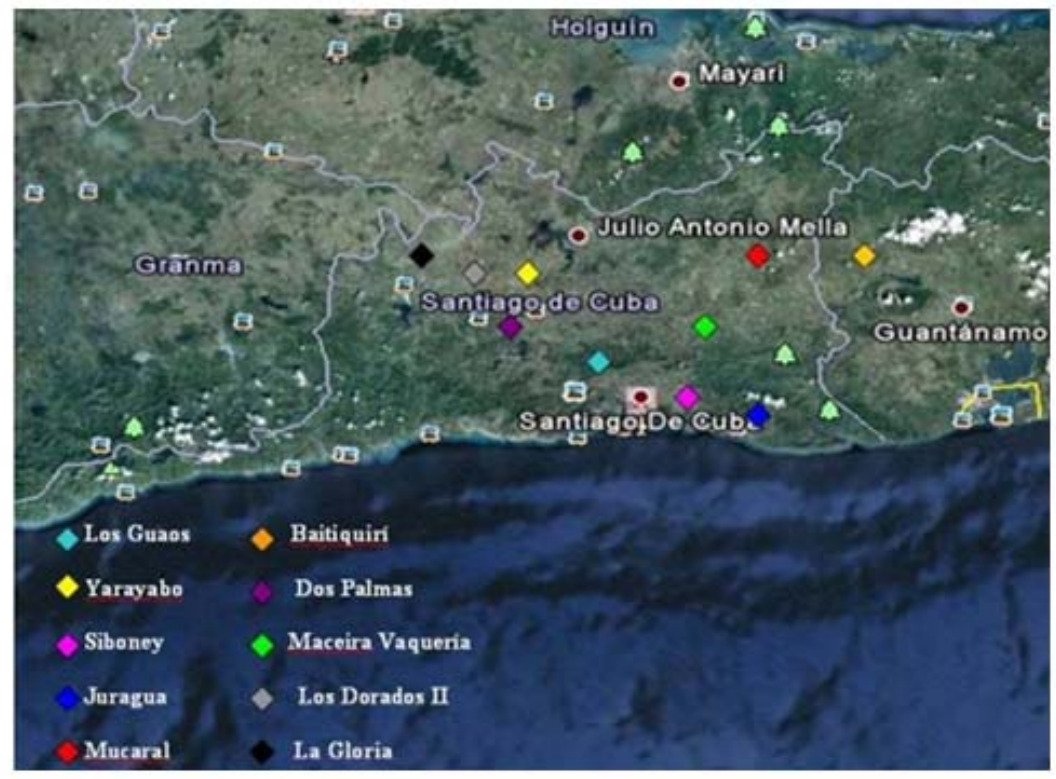

Figura 1. Ubicación de los yacimientos de materiales de la construcción de Santiago de Cuba.

Algunos yacimientos se explotan hace alrededor de 30 años con la finalidad de obtener materia prima para la construcción. El aprovechamiento de estos recursos, que por otra parte son imprescindibles, produce efectos negativos sobre el medio ambiente, fundamentalmente por los grandes movimientos de tierra para alcanzar las rocas y minerales, lo que provoca profundos cambios en las formas del terreno, con el consiguiente deterioro del paisaje, además de alteraciones sobre el suelo, la vegetación, el agua y 
otros factores ambientales. En la provincia las áreas afectadas por esta actividad minera ocupan alrededor de 43 ha (Tabla 1).

Tabla 1. Estado de la rehabilitación de los yacimientos de la industria de materiales de construcción de Santiago de Cuba

\begin{tabular}{|c|c|c|}
\hline Canteras & Material que se extrae & Area minada no recuperada (ha) \\
\hline Los Guaos & Porfirita & 11,73 \\
\hline Juraguá & Arena & 7,45 \\
\hline Maceira Vaquería & Arcilla & 0,97 \\
\hline Los Dorados & Arena y grava & 2,02 \\
\hline Dos Palmas & Arcilla & 1,15 \\
\hline Mucaral & Caliza & 2,07 \\
\hline Siboney & Caliza & 0 \\
\hline Yarayabo & Caliza & 16,77 \\
\hline Baitiquirí & Yeso & 0,53 \\
\hline La Gloria & Caliza & 0,15 \\
\hline Total & & 42,84 \\
\hline
\end{tabular}

\section{Ubicación y caracterización del yacimiento Los Guaos}

El yacimiento objeto de estudio se encuentra ubicado en la provincia de Santiago de Cuba a $5 \mathrm{~km}$ al oeste de la ciudad de Santiago.

Las coordenadas Lambert son:

$X: 559500-602500$

Y: $155000-157250$

El área se encuentra en explotación desde enero de 1978 (34 años), y sus plantas constituyen las principales abastecedoras de áridos en la provincia de Santiago de Cuba.

El yacimiento Los Guaos está dividido en tres zonas de laboreo: Guaos I, cuyas reservas están agotadas; Guaos II, que es el que se explota actualmente; y Guaos III, en fase de desarrollo.

\section{Relieve}

El relieve en la región se puede clasificar como semi-montañoso, cuyo origen se relaciona con procesos tectónicos y erosivos. Al este del yacimiento se presentan las cotas más altas con valores de $231 \mathrm{~m}$ sobre el nivel del mar. Las cotas mínimas son de $50 \mathrm{~m}$, lo que se aprecia al oeste donde el relieve es más ondulado.

\section{Hidrografía}

La red hidrografía la forman los ríos Guaos y Gascón y algunos arroyos. Estos ríos con una dirección norte-sur, son de carácter intermitente en época de estiaje. La fuerza erosiva de los ríos es limitada debido a la pendiente longitudinal suave que presentan.

\section{Clima}

El clima es tropical y seco con temperaturas mayores de $260 \mathrm{C}$, la mayor parte del año. La temperatura media más alta en el período es de 31oC y la 
media más baja es de $260 \mathrm{c}$. La humedad relativa oscila entre un 70 y un 75\%. La precipitación media anual varía entre los 800 a 1000 mm de lluvia.

\section{Propiedades químicas y físico-mecánicas de la roca}

El material que se extrae en la cantera es la porfirita que es de color grisverdosa, compacta, maciza en muchas partes con visible agrietamiento natural. La resistencia a la comprensión presenta un valor mínimo de 800 $\mathrm{kg} / \mathrm{cm}^{2}$ y máximo de $1200 \mathrm{~kg} / \mathrm{cm}^{2}$, un peso volumétrico con valores entre 2,55 y $2,61 \mathrm{~g} / \mathrm{cm}^{3}$ seco y saturado respectivamente, y una absorción de $2,8 \%$. El coeficiente de esponjamiento es de 1,5 a 1,8.

\section{Descripción del medio biológico (flora y fauna)}

En la zona se puede encontrar una gran variedad de plantas: Manguiphera indica (mango), Dichrostachys glomerata (marabú), Leucaena glauca (aroma blanca), Laurocerasus occidentalis (almendro), Comocladia dentada (guao), Xylopia obtusifolia (malagueta), Annona glabra (palo bobo), Dipholis salicifolia (almendrillo) y Annona montana (guanábana de loma).

Entre la variedad faunística se encuentran: Rana castebiana (rana toro), Anolis porcados (lagartija verde), Epicrates anzulyso (majá), Crapromys presiles (jutía conga), Chorostibon ricordii (zunzún), Mimus polygottos (sinsonte), Columbina passerina (tojosa), Catharter aura (aura tiñosa) y el Phyllonyenys poeys (murciélago).

Ninguna de las especies inventariadas está considerada como amenazada de extinción, y la fauna residente en el área no es muy abundante en mamíferos y no presenta características especiales que ameriten criterio de conservación.

\section{Demografía y economía}

La zona no está densamente poblada; existe un asentamiento poblacional donde las viviendas en línea general tienen un estado regular. Cerca del yacimiento se encuentran como objetos socioeconómicos: la Textilera "Celia Sánchez" y la fábrica de baldosas, las que constituyen fuente de empleo para los habitantes de esa zona. El nivel cultural de la población en sentido general es medio.

\section{DESARROLLO}

Para realizar un plan de recuperación de un área determinada, se deben conocer los factores ambientales (clima, geología, fauna, flora, hidrología, paisaje, etc.) y culturales (demografía, economía e historia) del entorno y del lugar a recuperar. También es importante conocer la problemática del lugar (si ha habido contaminaciones del suelo, del agua, si ha habido compactaciones, edificaciones, etc.).

A partir de los requerimientos de los usos y actividades a desarrollar en el espacio (futuras previstas o potenciales) y de los factores condicionantes y limitantes, se deben definir los "modelos de recuperación" a aplicar a cada unidad del espacio. Cada modelo de recuperación consiste, básicamente, 
en una imagen objetivo en términos de cobertura vegetal o características de la superficie del terreno.

El procedimiento de recuperación elaborado, consta de 5 etapas fundamentales y se tuvieron en cuenta 4 grupos de prácticas, entre las que mencionamos: prácticas edáficas, prácticas topográficas y geotécnicas, prácticas hídricas y prácticas ecológicas.

Las prácticas edáficas están relacionadas con el manejo y protección del suelo, recurso escaso y de gran importancia en la recuperación de áreas dañadas. Este tipo de prácticas incluye la remoción selectiva del suelo, acciones de prevención de contaminación por productos químicos y de prevención de la erosión, entre otras.

Las prácticas de carácter topográfico y geotécnico incluyen el remodelamiento del terreno afectado, tanto en escombreras como en frentes de excavación.

Las prácticas geotécnicas buscan la estabilidad física del área, mientras que las prácticas topográficas buscan lograr un área de forma armoniosa con su entorno o establecer condiciones geomorfológicas similares a las que tenía el área antes de la minería.

Las prácticas hídricas buscan la conservación de la cantidad y calidad de las aguas superficiales y subterráneas.

La colección, transporte y descarga final de las aguas fluviales son aspectos fundamentales para la estabilidad física del área recuperada, así como para la protección de los recursos hídricos superficiales. En relación a la conservación de las aguas subterráneas, se incluyen prácticas relativas a la disminución del nivel freático para la protección de estos acuíferos contra la presencia de sustancias contaminantes.

Las prácticas de carácter ecológico se refieren a acciones relativas al manejo de la fauna y la vegetación, donde se busca el establecimiento de una comunidad vegetal en áreas designadas de la mina y su entorno.

PROCEDIMIENTO PARA RECUPERAR LAS ÁREAS MINADAS DE LAS CANTERAS DE MATERIALES DE CONSTRUCCIÓN DE SANTIAGO DE CUBA

Considerando los diversos aspectos incluidos en la recuperación de áreas degradas por la minería, se especifican las etapas y procedimientos básicos que deben guiar el trabajo en el caso de las canteras de materiales de construcción (Figura 2). 


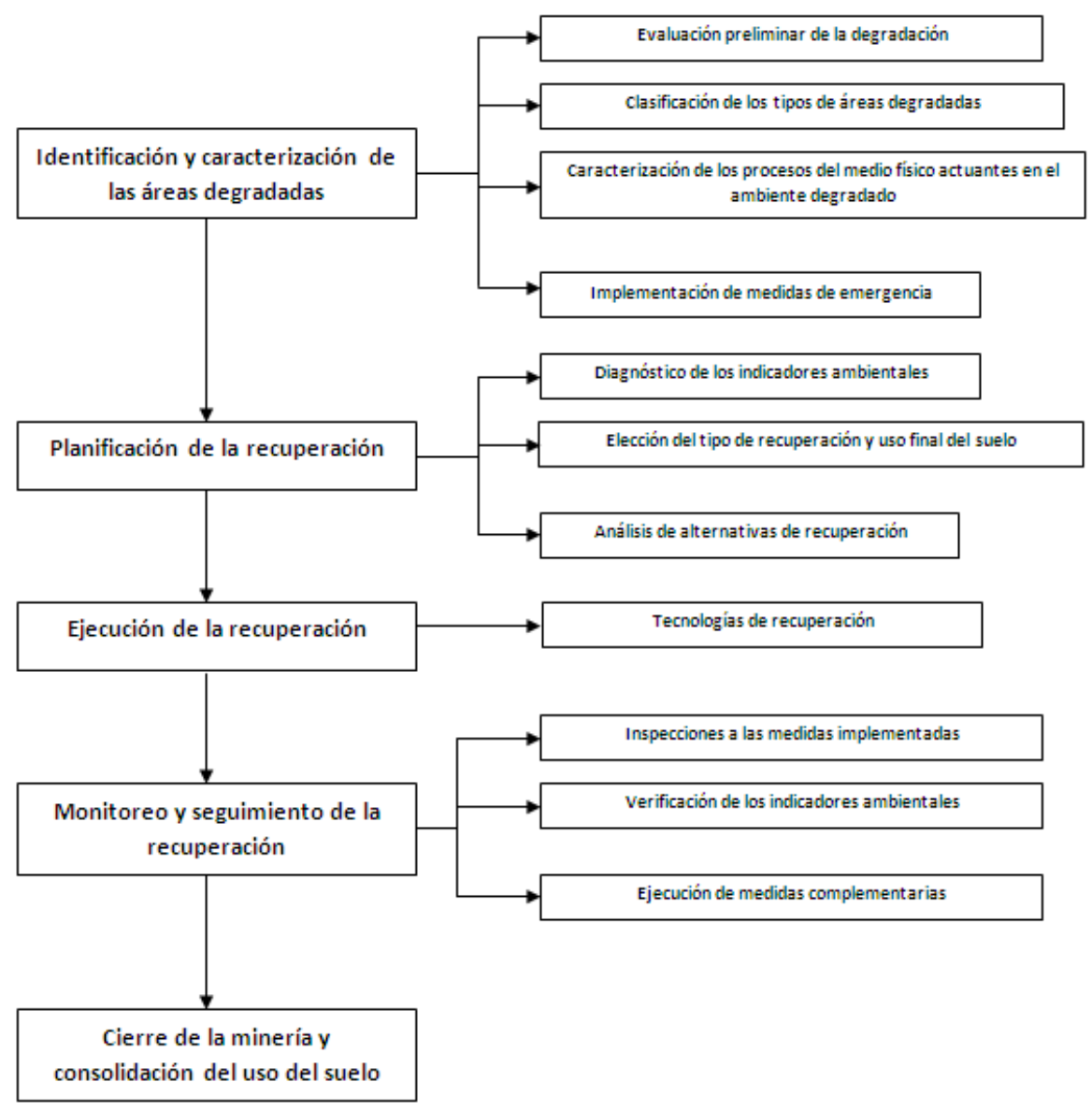

Figura 2. Procedimiento para la recuperación de áreas degradadas por la minería en canteras de materiales de construcción.

\section{Descripción del procedimiento elaborado}

Partiendo del procedimiento elaborado, a continuación se presenta la secuencia de actividades técnicas que deben componer el plan básico para determinadas áreas degradadas, que tienen como objetivo preliminar asegurar a corto plazo la estabilidad del medio ambiente de la zona y que dependen de una vinculación con un proyecto de rehabilitación futuro.

Se decidió aplicar el procedimiento a la Cantera Los Guaos debido a su situación ambiental relacionada con su explotación sin proyecto de rehabilitación hace 34 años, y a que es una de las canteras con más hectáreas dañadas por la minería en la Provincia. 


\title{
DISCUSIÓN Y ANÁLISIS DE LOS RESULTADOS
}

\author{
Identificación y caracterización de las áreas degradadas
}

\section{Evaluación preliminar de la degradación}

El primer paso del programa de recuperación de las áreas degradas de la cantera Los Guaos, es la evaluación preliminar de la degradación, a través de un cuidadoso diagnóstico de los procesos que allí actúan y de sus consecuencias ambientales. En visitas realizadas a la Cantera Los Guaos se realizó una evaluación preliminar de las áreas dañadas por la minería, se constató que los frentes en explotación presentan taludes muy inestables, con derrumbes parciales en algunos lugares y cambios en la topografía. En el área perteneciente al pasivo ambiental, el principal problema es la inestabilidad de los taludes. En las escombreras se evidencian grandes procesos erosivos y la propagación de polvo a la atmósfera, y en las obras de infraestructura el mayor impacto se observa en la contaminación por polvo de las plantas de preparación mecánica, así como el aumento del ruido y las vibraciones.

Cabe destacar que cerca del yacimiento Los Guaos, existe una comunidad llamada de la misma manera. En dicha comunidad no existe percepción del riesgo ambiental, a pesar de que algunos residentes refieren que se producen molestias por el ruido, el polvo y el impacto paisajístico, pero consideran importantes para la comunidad el mejoramiento de los caminos de acceso y la transportación y el aumento del empleo.

Finalmente, en esta evaluación preliminar se realizó un estudio preciso de la información disponible sobre todos los aspectos que pueden condicionar la recuperación de las zonas minadas, entre los que se pueden mencionar la topografía, la geología, los usos del suelo, las redes de comunicación, la flora, la fauna, etc., lo que permite la evaluación de la degradación. Lo anterior permitió clasificar por tipos las áreas degradadas (Tabla 3).

En la Cantera Los Guaos además del área que se encuentra en explotación, existe un área llamada Guaos I (Fotografía 2), que se abandonó por la inundación del río, dicha área constituye un pasivo ambiental, y en este sitio la recuperación de la vegetación es espontánea.

El área que se encuentra actualmente en explotación es Guaos II (Fotografía 1); en la Tabla 2 se muestran las principales actividades de la cantera, los factores que se ven afectados por dicha actividad y su impacto al medio ambiente (Risco, 2012). 
Tabla 2. Impactos ambientales producidos por la actividad minera en el yacimiento Guaos II

\begin{tabular}{|c|c|c|}
\hline Acción/Actividad & Factores que se afectan & Impacto \\
\hline Desbroce & $\begin{array}{l}\text { Suelo. } \\
\text { Flora y Fauna. } \\
\text { Paisaje. }\end{array}$ & $\begin{array}{l}\text { Remoción de la vegetación y } \\
\text { deforestación. } \\
\text { Alteración de la calidad visual. } \\
\text { Alteración del hábitat natural y } \\
\text { desplazamiento de la fauna. }\end{array}$ \\
\hline Destape & $\begin{array}{l}\text { Suelo. } \\
\text { Flora y Fauna. }\end{array}$ & $\begin{array}{l}\text { Aumento de los procesos erosivos. } \\
\text { Remoción de la vegetación y } \\
\text { deforestación. } \\
\text { Alteración del hábitat natural y } \\
\text { desplazamiento de la fauna. }\end{array}$ \\
\hline $\begin{array}{c}\text { Perforación, carga } \\
\text { y voladura }\end{array}$ & $\begin{array}{c}\text { Suelo. } \\
\text { Flora y Fauna. } \\
\text { Paisaje. } \\
\text { Atmósfera. } \\
\text { Aguas superficiales y subterráneas. }\end{array}$ & $\begin{array}{l}\text { Alteración de la calidad visual. } \\
\text { Emisión de gases y polvo a la } \\
\text { atmósfera. } \\
\text { Emisión de ruidos y vibraciones. } \\
\text { Alteración de la calidad del agua. } \\
\text { Remoción de la vegetación y } \\
\text { deforestación. } \\
\text { Alteración del hábitat natural y } \\
\text { desplazamiento de la fauna. }\end{array}$ \\
\hline $\begin{array}{l}\text { Formación de } \\
\text { escombreras }\end{array}$ & $\begin{array}{c}\text { Suelo. } \\
\text { Paisaje. } \\
\text { Atmósfera. }\end{array}$ & $\begin{array}{l}\text { Aumento de los procesos erosivos. } \\
\text { Alteración de la calidad visual. } \\
\text { Emisión de polvo a la atmósfera. }\end{array}$ \\
\hline $\begin{array}{c}\text { Preparación } \\
\text { mecánica }\end{array}$ & $\begin{array}{l}\text { Atmósfera. } \\
\text { Paisaje. } \\
\text { Agua. }\end{array}$ & $\begin{array}{l}\text { Alteración de la calidad visual. } \\
\text { Emisión de gases y polvo a la } \\
\text { atmósfera. } \\
\text { Emisión de ruidos y vibraciones. } \\
\text { Obstrucción y encenagamiento del } \\
\text { río. }\end{array}$ \\
\hline $\begin{array}{c}\text { Extracción y } \\
\text { transporte de } \\
\text { material }\end{array}$ & $\begin{array}{l}\text { Atmósfera. } \\
\text { Paisaje. } \\
\text { Suelo. } \\
\text { Agua. } \\
\text { Flora y Fauna. }\end{array}$ & $\begin{array}{l}\text { Alteración de la calidad visual. } \\
\text { Emiśón de gases y polvo a la } \\
\text { atmósfera. } \\
\text { Emisión de ruidos y vibraciones. } \\
\text { Alteración de la calidad del agua. } \\
\text { Obstrucción y encenagamiento del } \\
\text { río. } \\
\text { Alteración del nivel freático. } \\
\text { Remoción de la vegetación y } \\
\text { deforestación. } \\
\text { Alteración del hábitat naturaly } \\
\text { desplazamiento de la fauna. }\end{array}$ \\
\hline
\end{tabular}

\section{Clasificación de los tipos de áreas degradadas}

Sobre la base de los tipos de áreas degradadas (Yazbek, 2000) que se consideran para las canteras de materiales de construcción establecidas en la metodología, se propuso la siguiente clasificación:

Tabla 3. Clasificación de las áreas degradas en el yacimiento Los Guaos

\begin{tabular}{|c|c|}
\hline \multicolumn{2}{|c|}{ Tipos de areas degradadas } \\
\hline Areas explotadas & - Frentes de explotación y taludes. \\
\hline $\begin{array}{l}\text { Areas de depósitos de estériles y } \\
\text { desechos }\end{array}$ & - Escombreras. \\
\hline Areas de infraestructura & $\begin{array}{l}\text { - Area de almacenaje y expedición de mineral. } \\
\text { - Vías de circulación. } \\
\text { - Talleres de máquinas y equipos. }\end{array}$ \\
\hline
\end{tabular}

\section{Caracterización de los procesos del medio físico actuantes en el medio ambiente degradado}

Dentro de las alteraciones sobre el recurso tierra las primeras modificaciones se producen sobre el suelo, que como resultado del destape 
se eliminan totalmente. Posteriormente durante la extracción del material por la acción de la explosión y voladura se afecta el suelo y el medio ambiente en general, lo cual trae consigo que se produzcan cambios en el relieve donde se transforma la topografía y se altera generalmente de forma radical el drenaje natural, quedando los cortes mineros, un nuevo relieve denominado antropotecnógeno.

Las alteraciones a las aguas superficiales se producen por los cambios en su composición físico-química, producto del arrastre de partículas por erosión. El agua subterránea se ve sometida a cambios en su composición físico-química por infiltración, afectándose también los niveles freáticos. Comúnmente en el relieve irregular creado se forman acumulaciones de agua, verdaderas lagunas, que tienen carácter tanto permanente como temporal. Las estructuras creadas después de la extracción cambian el entorno de las explotaciones, y la recuperación de las condiciones originales resultan muy difíciles.

En las superficies denudadas por destape, aparecen los procesos de deflación y por tanto de contaminación del aire, así como también en ellas ocurren procesos erosivos de arrastres de partículas que contaminan las aguas superficiales. Estos procesos negativos son de carácter temporal.

El escurrimiento y la red de drenaje natural se alteran generalmente, provocándose un intenso arrastre de partículas por erosión desde estas superficies, que contaminan las aguas superficiales.

En las escombreras al igual que en las superficies de extracción de minerales, se evidencian procesos erosivos intensos con formación de surcos, cárcavas y deslizamientos provocando la contaminación de las aguas de los ríos y sus efluentes. El efecto de la erosión tiene carácter temporal en estas superficies.

Las superficies ocupadas por residuales mineros son áreas que están afectadas por la contaminación del suelo producto de la deposición de materiales, también en ellas se desarrollan los procesos erosivos, sedimentación y compactación.

En el área del pasivo ambiental (Guaos I) se presentan cambios en el relieve, la topografía y en los drenajes superficiales.

En la Tabla 4 se muestran los procesos del medio físico que producen impactos en cada una de las áreas degradas que tiene la Cantera Los Guaos.

Tabla 4. Procesos que producen impactos en el área degrada

\begin{tabular}{|c|c|}
\hline Tipo de área degradada & $\begin{array}{c}\text { Procesos del medio físico que } \\
\text { producen impactos }\end{array}$ \\
\hline Frentes de explotación Guaos I & Erosión, compactación. \\
\hline Frentes de explotación Guaos II & $\begin{array}{c}\text { Erosión, sedimentación. } \\
\text { Alteraciones a las aguas superficiales. }\end{array}$ \\
\hline Escombrera & Erosión, sedimentación y compactación. \\
\hline $\begin{array}{c}\text { Area de almacenaje y expedición de mineral. } \\
\text { Vías de circulación. }\end{array}$ & $\begin{array}{c}\text { Contaminación del suelo. } \\
\text { Compactación. }\end{array}$ \\
Talleres de máquinas y equipos. & Alteraciones a las aguas superficiales. \\
\hline
\end{tabular}




\section{Medidas de emergencia para la recuperación}

De acuerdo a la caracterización efectuada en esta primera etapa, son necesarias medidas inmediatas y urgentes para el mantenimiento de las zonas minadas:

- La revegetación de taludes y caminos internos.

- Extracción de bloques de rocas inestables en zonas minadas.

- Protección y manejo de la vegetación remanente.

- La inducción y la revegetación espontánea.

- Manejo de sectores abandonados en el sentido de favorecer la recuperación espontánea.

\section{Planificación de la recuperación}

\section{Diagnóstico de los indicadores ambientales}

En el minado antiguo (Guaos I), no se establecieron indicadores ambientales, debido a que los procesos ambientales actuantes en el medio degradado son leves y ha habido una colonización espontánea de la vegetación.

En el área de explotación actual (Guaos II), se tuvieron en cuenta tres indicadores ambientales: la concentración de polvo en el aire, el nivel de ruido en la cantera, y la contaminación de las aguas.

Los principales contaminantes del aire son las nubes de polvo causadas por el tráfico de camiones por caminos no pavimentados, por la actividad de perforación y voladura, por las escombreras denudadas y por la producción de la planta de preparación mecánica por vía seca, que producen partículas en suspensión en función de su granulometría, humedad y vientos predominantes, que son transportadas a distancias variables, y que en temporadas de estiaje o sequía se incrementan en gran medida, lo que disminuye la calidad del recurso natural aire.

Para la determinación de la concentración de polvo sedimentable se utilizó el método de Rigelmann, efectuando el muestreo en dos periodos (lluvia y seca). Se colocaron frascos de cristal de igual área durante 24 días y finalmente se obtuvo la concentración de polvo sedimentable por áreas. Como resultado se obtuvo que en 4 puntos la concentración de polvo se encontró por debajo de la norma permisible, lo que caracteriza este impacto como uno de los de mayor significación.

\section{Elección del tipo de recuperación y uso final}

En entrevistas a autoridades de planificación física en la provincia de Santiago de Cuba, se planteó que el uso propuesto para las áreas del minado antiguo (Guaos I) en el yacimiento Los Guaos será recreativo intensivo (campamento pioneril), para lo cual cumple con los parámetros suficientes y necesarios para este uso (Tabla 5).

Para la elección del uso final de la cantera, se plantea por el autor darle un uso final forestal al área que actualmente se encuentra en explotación 
(Guaos II), para lo cual se tuvieron en cuenta los aspectos necesarios que deben cumplir estos terrenos para este uso en función del tipo de minería.

Tabla 5. Uso final de las áreas en explotación y del minado antiguo

\begin{tabular}{|c|c|c|}
\hline Tipo de área degradada & Uso final & Aspectos necesarios \\
\hline $\begin{array}{c}\text { Zonas de minado antiguo } \\
\text { (Fotografía 2) }\end{array}$ & $\begin{array}{l}\text { Recreativo intensivo } \\
\text { (campamento pioneril) }\end{array}$ & $\begin{array}{l}\text { - Buena estabilidad de los taludes. } \\
\text { - Accesos. } \\
\text { - Buenas propiedades geotécnicas del } \\
\text { suelo. } \\
\text { - Proximidad a núcleos urbanos. } \\
\text { - Medidas de seguridad para los } \\
\text { usuarios. } \\
\text { - Buen acondicionamiento de la } \\
\text { excavación. }\end{array}$ \\
\hline $\begin{array}{l}\text { Zonas en explotación } \\
\text { (Fotografía 1) }\end{array}$ & Forestal & $\begin{array}{l}\text { - Pendientes moderadas. } \\
\text { - Suelo fértil bien reconstituido. } \\
\text { - Buen sistema de drenaje. } \\
\text { - Selección de especies. }\end{array}$ \\
\hline
\end{tabular}

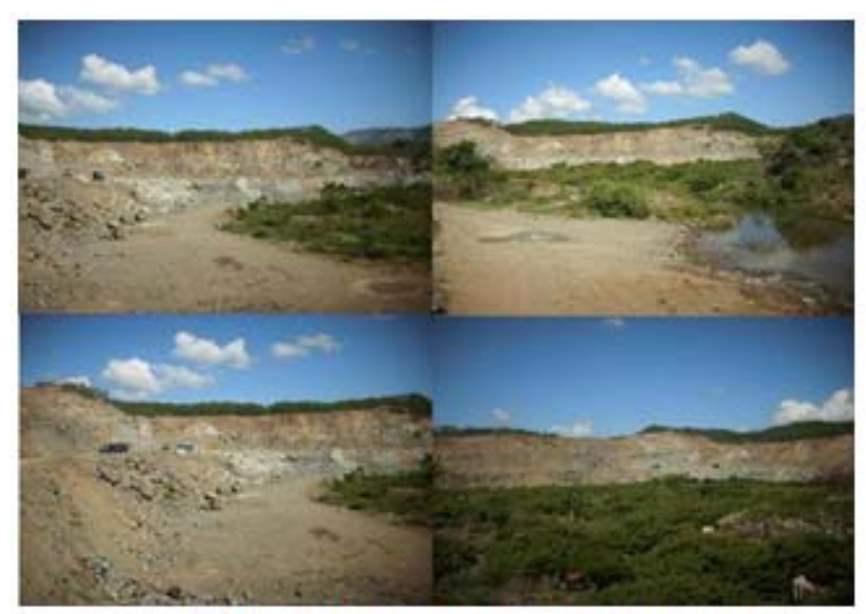

Fotografía 1. Zona en explotación.

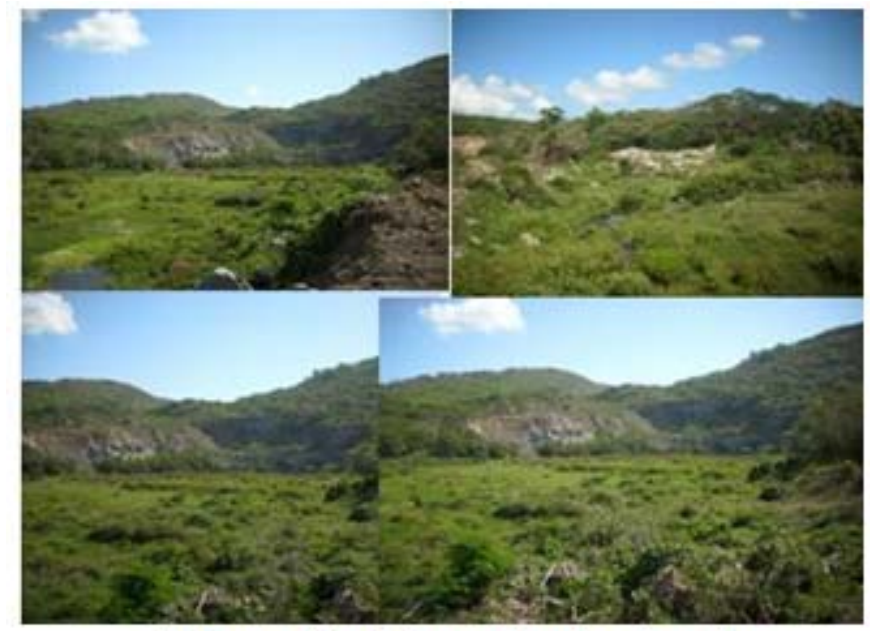

Fotografía 2. Zona de minado antiguo. 


\section{Ejecución de la recuperación}

\section{Medidas de recuperación en el área de la zona del minado antiguo}

El uso futuro que se le propone a la zona del minado antiguo (Fotografía 2) es el recreativo intensivo (campamento pioneril), ya que es un área suburbana con cercanía de una comunidad. Estos terrenos abandonados pueden ser adecuados para desarrollar diferentes actividades recreativas y deportivas, especialmente para los niños. Para darle este uso final, se deben tomar una serie de medidas que hay que integrar al proyecto de explotación para obtener un adecuado uso final de la explotación.

\section{Medidas de recuperación en el área de explotación de la cantera}

El uso futuro que se le propone al área que actualmente se encuentra en explotación (Fotografía 1) es forestal; aunque normalmente su instauración es más cara, en este caso resulta más económico que el uso agrícola, ya que se aprovechará la condición de que es necesario liberar el área del indeseado marabú, y fomentar la siembra de árboles maderables y frutales. Los resultados de este uso se podrán alcanzar a mediano y largo plazo.

Para darle este uso final, habrá que tomar las medidas que hay que integrar al proyecto de explotación para obtener un adecuado uso final del área degradada.

También, para lograr la recuperación completa del yacimiento se deben cumplir una serie de medidas en las demás áreas del yacimiento, entre las que podemos mencionar: medidas de recuperación en el área de la escombrera y medidas de recuperación en áreas de almacenaje y expedición de mineral, vías de circulación, talleres de máquinas y equipos.

\section{Monitoreo y seguimiento de la recuperación}

En esta fase se realiza el monitoreo de la calidad ambiental de la recuperación y se da el seguimiento de la efectividad de la misma, realizándose estudios de supervivencia de flora, así como la toma de muestra de las aguas expuestas a tratamiento para conocer si cumplen con los parámetros ambientales de vertimiento establecidos.

\section{Inspecciones a las medidas implementadas}

a) Monitoreo de las aguas superficiales.

b) Monitoreo del suelo.

c) Monitoreo de la biodiversidad.

d) Monitoreo del paisaje.

\section{Verificación de los indicadores ambientales}

Esta verificación se realizará por entidades acreditadas y avalados por estudios de evaluación de impactos, estudio de supervivencias y estudio de la calidad ambiental del agua. A partir de los resultados obtenidos se verificará el comportamiento de los indicadores ambientales. Serán chequeados los parámetros de $\mathrm{pH}$, sólidos sedimentables y la presencia de vida acuática en los efluentes recuperados; respecto al suelo se analizará la estabilidad de los taludes, así como la revegetación en taludes y en 
escombreras; el relieve se analizará cómo este se va integrando al paisaje con la aplicación de todas las medidas recuperadoras.

\section{Implementación de medidas complementarias}

Después de verificados los indicadores ambientales, si existe algún indicador que no responda a las expectativas esperadas serán implementadas medidas complementarias para lograr que el uso final propuesto se cumpla sin contratiempos.

\section{Cierre de la minería y consolidación del uso del suelo}

El objetivo de la actividad de cierre, en la Cantera Los Guaos, será asegurar para la etapa de abandono la estabilidad física y química de las instalaciones y estructuras, así como también la del uso del suelo, para evitar daño a las personas y al medio ambiente a largo plazo.

Aquí se proponen una serie de medidas que, integradas con las medidas de recuperación aplicadas en cada área degradada, lograrán que en la cantera se logre un cierre adecuado.

\section{CONCLUSIONES}

- El procedimiento elaborado para la recuperación de las áreas afectadas por la minería en las canteras de materiales de construcción de la provincia de Santiago de Cuba, permitirá el mejoramiento de la calidad ambiental, social y económica en las zonas afectadas por la actividad minera y el logro de una minería responsable.

- Se aplicó el procedimiento en la Cantera Los Guaos de la provincia de Santiago de Cuba, y se eligió el uso recreativo intensivo para las áreas del minado antiguo, y forestal para las áreas que actualmente se encuentran en explotación.

- La aplicación del procedimiento en la Cantera Los Guaos evidenció la efectividad del mismo, y la posibilidad de uso en las demás canteras de la Provincia.

\section{REFERENCIAS}

- Asamblea Nacional del Poder Popular de la República de Cuba. Ley 76. Ley de Minas. 1994. La Habana.

- _ L Ley 81. Ley del Medio Ambiente. 1997. La Habana.

- Carbonell Veja, F. (2003). Evaluación del impacto ambiental que se genera durante la explotación del yacimiento la Yaya y en el proceso industrial de la calera (Tesis de Maestría).

- Parrota, J.A. y Knowles, O.H. (2001). Restoring tropical forests on lands mined for bauxita: Examples from the Brazilian. Ecological Engineering, 17, 219-239 
- Quesada, R.W. (2008). Situación actual y perspectiva de la explotación de yacimientos de materiales de construcción. ISMM. Moa. (Inédito). 20 p.

- Risco, M.A. (2012). Recuperación de áreas minadas de canteras de materiales de construcción de Santiago de Cuba (Tesis de Maestría).

- Yazbek, B.O. (2000). Recuperación de áreas degradadas por la minería en regiones urbanas. II Curso Internacional de aspectos geológicos de protección ambiental. Campinas, São Paolo, Brasil.

1. Máster en Minería. Profesor Auxiliar. Instituto Superior MineroMetalúrgico de Moa. Facultad Geología-Minería. Departamento de Minería.amontes@ismm.edu.cu

2. Doctora en Ciencias Económicas. Profesora Titular. Instituto Superior Minero-Metalúrgico de Moa. Facultad Geología-Minería. Departamento de Minería. mulloac@ismm.edu.cu

Para citar este artículo: Montes de Oca-Risco, A. \& Ulloa-Carcassés, M. (2013). Recuperación de áreas dañadas por la minería en la Cantera Los Guaos, Santiago de Cuba, Cuba. Revista
Luna
Azul,
37 ,
74-88.
Recuperado
de

http://lunazul.ucaldas.edu.co/index.php?option=content\&task=view\&id=846 